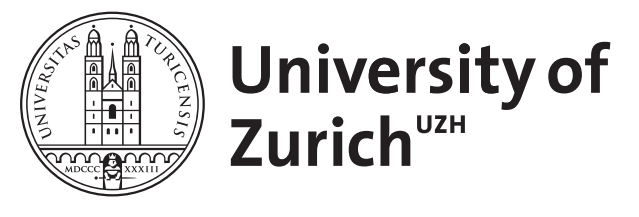

\title{
Akute Gefäßerkrankungen in der Gastroenterologie
}

\author{
Grubhofer, F ; Pecoraro, F ; Gilhofer, T S ; Gubler, C ; Lurje, G ; Schneider, P M ; Lachat, Mario
}

\begin{abstract}
Acute gastroenterologic vascular emergencies are common situations in emergency departments and the clinical consequences range from trivial to life-threatening. Only the early recognition of these symptom patterns and prompt use of the appropriate diagnostic tools lead to a correct diagnosis with subsequent potentially life-saving treatment. To decrease the high mortality rate of acute mesenteric ischemia (50\%), aorto-enteric fistula (30-40\%), visceral artery aneurysms (10-100\%) and Budd-Chiari syndrome new strategies with an endovascular approach are gaining importance and are partially replacing established diagnostic and therapeutic algorithms. This article provides a review of the diagnosis and therapy of these gastroenterologic emergency situations.
\end{abstract}

DOI: https://doi.org/10.1007/s11377-011-0580-7

Posted at the Zurich Open Repository and Archive, University of Zurich

ZORA URL: https://doi.org/10.5167/uzh-71450

Journal Article

Published Version

Originally published at:

Grubhofer, F; Pecoraro, F; Gilhofer, T S; Gubler, C; Lurje, G; Schneider, P M; Lachat, Mario (2012). Akute Gefäßerkrankungen in der Gastroenterologie. Der Gastroenterologe, 7(2):111-120.

DOI: https://doi.org/10.1007/s11377-011-0580-7 
Gastroenterologe $2012 \cdot 7: 111-120$

DOI 10.1007/s11377-011-0580-7

๑) Springer-Verlag 2012

\section{Redaktion}

M. Fried, Zürich

W. Schepp, München
F. Grubhofer ${ }^{1}$. F. Pecoraro ${ }^{2}$. T.S. Gilhofer ${ }^{3}$. C. Gubler ${ }^{4}$. G. Lurje ${ }^{1}$.

P.M. Schneider ${ }^{1} \cdot$ M. Lachat $^{5}$

${ }^{1}$ Klinik für Viszeral- und Transplantationschirurgie, UniversitätsSpital Zürich

${ }^{2}$ Azienda Ospedaliera Universitaria Policlinico Paolo Giaccone , Universität Palermo

${ }^{3}$ Klinik für Innere Medizin, Spital Lachen

${ }^{4}$ Klinik für Gastroenterologie und Hepatologie, UniversitätsSpital Zürich

${ }^{5}$ Klinik für Herz- und Gefässchirurgie, UniversitätsSpital Zürich

\section{Akute Gefäßerkrankungen in der Gastroenterologie}

\begin{abstract}
Vaskuläre Notfälle in der Gastroenterologie haben aufgrund der zunehmenden Lebenserwartung in der westlichen Welt und der damit verbundenen erhöhten Inzidenz von pathologischen Gefäßwandveränderungen weiter an Bedeutung zugenommen. Um die seit beinahe 30 Jahren konstant hohe Mortalitätsrate zu senken, ist das adäquate Management der erstbetreuenden Ärzte eine essenzielle Voraussetzung.
\end{abstract}

\section{Akute mesenteriale Ischämie \\ Epidemiologie}

Die Inzidenz von atherosklerotischen Gefäßwandveränderungen in mesenterischen Blutgefäßen beträgt bei Männern im 7. Lebensjahrzehnt etwa $20 \%$ [1]. Acosta et al. analysierten im Zeitraum von 1979 bis 1982 die Inzidenz der akuten mesenterialen Ischämie anhand von Operations- und Autopsieberichten in der Bevölkerung von Malmö (Schweden). Die durchschnittliche Inzidenz betrug hierbei 12,9/100.000 Personen pro Jahr. Bei $67,2 \%$ der Patienten war ein akuter Verschluss der A. mesenterica superior, bei 15,7\% eine Mesenterialvenenthrombose, bei $15,4 \%$ eine nichtokklusive mesenteriale Ischämie die zugrunde liegende Ursache. Bei 1,7\% konnte der Grund nicht abschließend geklärt werden [2].

Kardioembolische Ereignisse führten am häufigsten zur akuten Mesenterial- ischämie [3], und bei mehr als einem Drittel der Patienten konnte in der Anamnese eine vorangegangene Thromboembolie festgestellt werden. Die A. mesenterica superior stellt aufgrund ihrer Form und anatomischen Lage das häufigste Ziel von mesenterialen Emboli dar. In den Fällen, bei denen es zu Verschlüssen der kollateralen Blutversorgung kommt, ist häufig eine ausgedehnte Darmischämie zu beobachten, wobei nur $20 \%$ aller betroffenen Patienten Symptome einer Angina intestinalis zeigen.

Die nichtokklusive Mesenterialischämie nimmt eine Sonderstellung als Ursache für die akute ein. Diese Patienten präsentieren sich mit Verschlüssen des Viszeralarterienbaums und ischämischem Darm. Sie leiden meistens an einer systemischen Grunderkrankung, die ein enterales Perfusionsdefizit zur Folge hat. Als solches kann eine nichtokklusive Mesenterialischämie auch durch ein abdominelles Kompartmentsyndrom bei rupturierten abdominellen Aortenaneurysmen verursacht werden.

\section{Klinische Symptomatik}

Es gibt keine spezifischen klinischen Anzeichen oder Symptome einer Mesenterialischämie. Allgemein zeigen sich die Patienten mit Bauchschmerzen, Azidose, Diarrhö, Sepsis oder gastrointestinaler Blutung. Das subjektiv starke Schmerzempfinden steht häufig im Gegensatz zur klinischen Untersuchung, bei der das
Abdomen noch weich und nur diskret druckempfindlich ist [4].

$\triangle$ Eine ausgeprägte Darmischämie hat einen fulminanten Beginn ohne prodromale Symptomatik.

Der zeitliche diagnostische Aufwand beeinflusst die Prognose wesentlich. Wird die Diagnose innerhalb der ersten 24 Stunden gestellt, so ist eine Überlebensrate von ca. $50 \%$ zu erwarten. Danach sinkt die Überlebenswahrscheinlichkeit signifikant auf ca. 30\%. Bei Patienten mit vorbestehender thrombotischer Gefäßerkrankung kann die akute Mesenterialischämie auch mit subakuter Symptomatik auftreten [5].

Es bestehen keine spezifischen Laborparameter, die explizit auf eine akute Mesenterialischämie hinweisen. Die Leukozytose ist wie die metabolische Azidose ein häufiger und zugleich unspezifischer Befund.

\section{Diagnostik}

Die Diagnose stützt sich meist auf die computertomographische Angiographie (CTA) oder auf den intraoperativen Befund.

- Die Sensitivität der CTA liegt bei $100 \%$, die Spezifität bei $90 \%$, mit einem negativen Vorhersagewert von $100 \%$ und einem positiven Vorhersagewert von 96\% [6, 7]. In der Studien-Bevölkerungsgruppe aus Malmö 


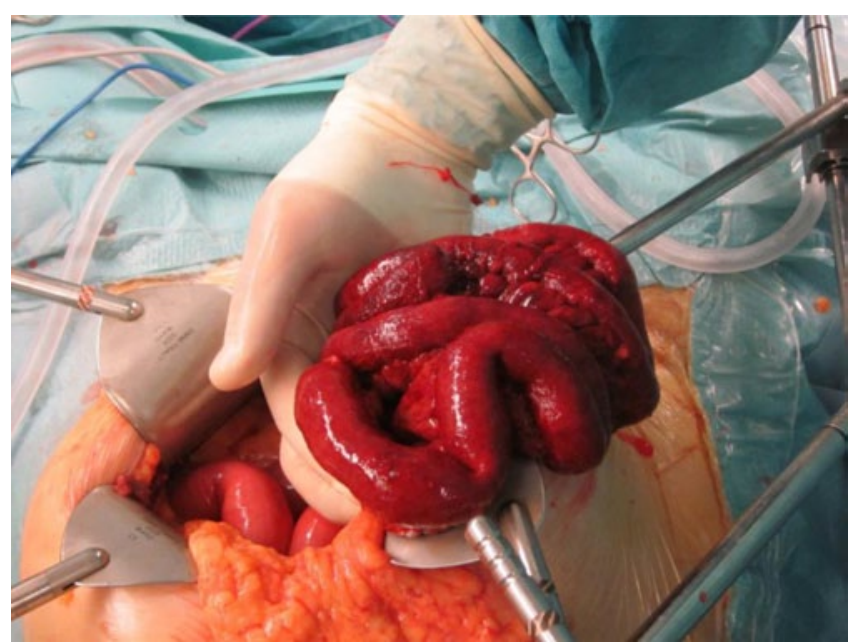

Abb. 14 Akute Mesenterialischämie des distalen Jejunums $24 \mathrm{~h}$ nach Symptombeginn

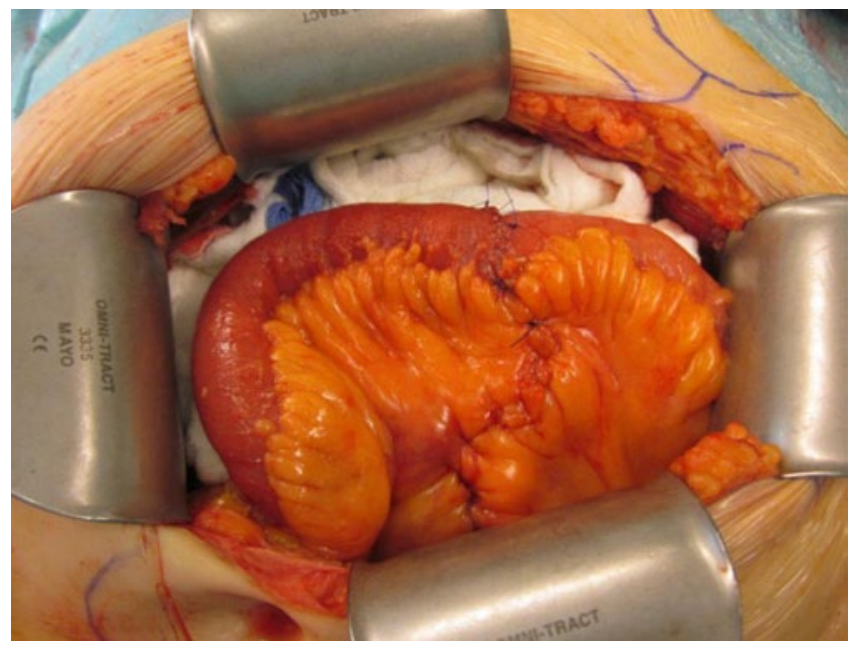

Abb. $2<$ Segmentresektion und Wiederherstellung der Dünndarmkontinuität

konnte nachgewiesen werden, dass die Verwendung der CTA in der präoperativen Diagnostik eine statistisch signifikante Senkung der Mortalitätsrate zur Folge hat [2].

- Die Röntgenübersichtsaufnahme ist bei $25 \%$ der Patienten unauffällig. Hier können sich im Frühstadium Zeichen eines Ileus, im Spätstadium Zeichen einer Pneumatosis intestinalis zeigen.

- Die abdominelle Sonographie stellt eine nichtinvasive diagnostische Möglichkeit mit hoher Treffsicherheit für die Darstellung von Gefäßverschlüssen des Truncus coeliacus und der A. mesenterica superior dar. Sie hat sich derzeit aber noch nicht als diagnostisches Verfahren etablieren können, da es sich um eine anwenderabhängige Untersuchung handelt und ein erfahrener Untersucher nicht immer schnell genug zur Verfügung steht.

- Im Rahmen der Behandlung der akuten Mesenterialischämie wird die Angiographie derzeit sowohl als invasives als auch diagnostisches Hilfsmittel bei endovaskulären Eingriffen verwendet.

- Die Vorteile der Magnetresonanzangiographie (MRA) liegen in der anatomischen Darstellung mit gleichzeitiger funktioneller Erfassung der Ischämie.

- Die Laparoskopie wird ebenfalls als diagnostische Maßnahme verwendet, allerdings limitiert die eingeschränkte Übersicht das diagnostische Potenzial.

Die Diagnosestellung der akuten mesenterialen Ischämie bleibt somit weiterhin eine Herausforderung - was sich in der
Tatsache widerspiegelt, dass nur ein Drittel aller betroffenen Patienten korrekt diagnostiziert wird $[8,9]$.

\section{Therapie}

\section{Medikamentöse Therapie}

Durch die Einführung der Angiographie in den 70er Jahren konnte die Mortalität der akuten Mesenterialischämie deutlich gesenkt werden; seither kam es aber zu keinem weiteren Mortalitätsrückgang mehr [2]. Die initiale Therapie stellt die Wiederherstellung der Elektrolytbalance, eine ausreichende antibiotische Therapie und - wenn keine Kontraindikationen dagegen sprechen - eine systemische Heparin-Infusionstherapie dar. Bei Verdacht auf Sepsis oder bei Organversagen sollten keine Vasopressoren eingesetzt werden [10].

Für die nichtokklusive Mesenterialischämie gibt es derzeit kein etabliertes Therapiekonzept; die aktuelle Therapie besteht aus Vasodilatatoren, Glukagon, Antibiotika und Nitroglyzerin.

\section{Chirurgie}

Sobald die Diagnose gestellt ist, ist eine notfallmäßige Laparotomie indiziert. Sollte sich ein ausgeprägter nekrotischer Befund zeigen, so ist eine Resektion des betroffenen Darmabschnitts unumgänglich (• Abb. 1, 2). Grundsätzlich sollte aber die Darmresektion nur dann der Revaskularisation vorangehen, wenn eine diffuse Darmnekrose, Darmperforation oder eine fortgeschrittene peritoneale Entzündung vorliegt.

\section{) Bei ausgeprägtem nekrotischem Befund ist eine Resektion des betroffenen Darmabschnitts unumgänglich}

Bei embolisch bedingter akuter Mesenterialischämie ist eine Embolektomie mittels Ballonkatheter indiziert. Im Gegensatz dazu wird bei der thrombotisch bedingten die anterograde Bypassanlage durchgeführt. Die Vorgehensweise bei der Bypassanlage ist zum einen von der okkludierenden Läsionslokalisation und zum anderen vom Operateur abhängig. 
Hier steht eine Anzeige.

黛 Springer 


\section{Symptome und Diagnostik}

Wie auch bei der arteriell bedingten Mesenterialischämie sind die Symptome bei der Mesenterialvenenthrombose vage und unspezifisch. Patienten äußern häufig einen diffusen Bauchschmerz, der sich im Durchschnitt 7-10 Tage konstant präsentiert. Es sind vor allem die Symptome der vorbestehenden oder koexistenten Krankheitsbilder wie Sepsis oder Pankreatitis, die zur Diagnose hinführen. Ein positiver Hämokkult-Test oder ein blutiges peritoneales Punktat weisen auf eine bereits fortgeschrittene Erkrankung hin.

Besteht der Verdacht auf eine Mesenterialvenenthrombose, so sollte eine Evaluation bezüglich eines etwaigen Protein-C-, Protein-S- oder Antithrombin-III-Mangels [20] stattfinden. Zudem sollten die Prothrombinzeit, die aktivierte partielle Prothrombinzeit, die AntiphospholipidAntikörper und die Plättchenaggregation untersucht werden. Die spezifischsten Laborwerte sind Leukozytose und Azidose. Leider treten diese laborchemischen Zeichen einer Darmischämie erst im späten Krankheitsverlauf auf.

Die Computertomographie und die Angiographie bewährten sich in verschiedenen Studien als gleichwertig. Dennoch wird von vielen Experten und Zentren die Computertomographie als das diagnostische Mittel der Wahl angesehen.

Trotz aller diagnostischen Hilfsmittel wird die Diagnose meist erst durch die Laparotomie oder die Autopsie gestellt. Kann die Diagnose vorher gestellt werden, so ist bei hämodynamisch instabilen $\mathrm{Pa}$ tienten oder Patienten mit Zeichen einer Peritonitis oder Darmischämie eine Notfalllaparotomie indiziert [7].

\section{Aortointestinale Fistel}

\section{Definition und Symptome}

Die aortointestinale Fistel ist eine seltene, aber lebensbedrohliche Komplikation, die im Spontanverlauf eines Aortenaneurysmas (primäre Form) oder postoperativ nach prothetischem Ersatz (sekundäre Fistel) auftreten kann. Die klassische Symptomentrias besteht aus gastrointestinaler Blutung in Kombination mit Bauchschmerz und tastbarer, pulsierender ab- domineller Resistenz oder vorbekanntem abdominellen Aortenaneurysma. In 94\% der Fälle ist ein Aortenaneurysma Ursache für eine aortointestinale Fistel [21].

\section{Epidemiologie und Pathogenese}

Die Inzidenz primärer Fisteln liegt zwischen 0,04 und 0,07\% bzw. zwischen 0,36 und 1,6\% für sekundäre Fisteln nach Aortenoperation [22]. Die Entstehung einer aortointestinalen Fistel lässt sich durch die konstante pulsatile Bewegung des Aneurysmas erklären, die zu einer zunehmenden - zumeist duodenalen - Erosion führt. In ca. 50\% der Fälle findet man die aortointestinale Fistel zwischen Aorta und Duodenum. Die initiale Blutung limitiert sich meist durch die spontane Thrombusbildung im Fistelgang. Der Zeitrahmen, in dem sich das schützende Blutgerinnsel auflöst bzw. wegbricht, reicht von wenigen Stunden bis zu mehreren Monaten. Dennoch zeigt sich in $30 \%$ der Fälle eine massive Blutung $6 \mathrm{~h}$ nach der Initialblutung [23].

\section{Diagnostik}

Mit der Gastroduodenoskopie lässt sich in $25-80 \%$ eine aortointestinale Fistel aufdecken [22]. Die höchste Sensitivität weist die CTA mit einer Detektionsrate von 93\% auf [24]. Aortale Lufteinschlüsse und intestinale Kontrastmittelanreicherungen gelten als pathognomische CTA-Befunde.

Der Einsatz der Arteriographie wird trotz des hohen Potenzials, eine aortointestinale Fistel aufzudecken, kontrovers betrachtet, da durch die diagnostische Maßnahme per se ein bestehender Fistelthrombus disloziert werden kann. Somit erfolgt eine Arteriographie zumeist nur im Anschluss an eine CTA, in der keine Blutungsquelle nachweisbar war [22].

\section{Therapie}

Die Blutstillung, Infektsanierung sowie Wiederherstellung der Blutflusskontinuität steht therapeutisch im Vordergrund [22]. Die 30-Tage-Mortalität einer offenen Operation zur Versorgung einer Primärfistel liegt zwischen 30 und 40\% [21]. Die endovaskuläre Therapie hat eine geringere Mortalitätsrate und wird bevorzugt einge- setzt, um eine schnelle Blutstillung zu gewährleisten [25]. Allerdings kann durch die endovaskuläre Therapie kein infiziertes Gefäßgewebe oder Prothesenmaterial entfernt werden, so dass es sich empfiehlt, bei hämodynamisch stabilen Patienten mit guter Lebenserwartung und wenig Komorbiditäten eine extraanatomische Bypassimplantation mit Exzision der infizierten Areale und Darmwandsanierung anzustreben. Dennoch ist auch der endovaskuläre Therapieansatz mit einer hohen Mortalitätsrate verbunden.

Neben der chirurgischen oder endovaskulären Therapie ist die begleitende antibiotische Therapie ein wichtiger Eckpfeiler.

\section{Aneurysmen viszeraler Arterien}

\section{Epidemiologie}

Aneurysmen viszeraler Arterien (AVA) stellen eine seltene, aber klinisch relevante Erkrankung dar. Insgesamt sind etwa 2500 Fälle weltweit beschrieben worden. Das renale Arterienaneurysma hat eine Inzidenz von 0,01-0,09\% [26]. Zusammen mit weiteren AVA ergibt sich eine Inzidenz von $0,1-2 \%$. Neben der A. renalis ist die A. lienalis das am zweithäufigsten betroffene Gefäß (• Abb. 3, [27]). Frauen sind von lienalen Aneurysmen um den Faktor 4:1 häufiger betroffen als Männer. Neben pathologischen Gefäßwandveränderungen können auch erworbene Ursachen wie Akzelerationstraumata bei Verkehrsunfällen, Drogenmissbrauch (Amphetamine) oder diagnostische endovaskuläre Eingriffe zu AVA führen. Die Mortalitätsrate der notfallmäßigen gefäßchirurgischen Therapie liegt zwischen 10 und $100 \%$, wobei sich eine besonders hohe Mortalitätsrate für das Aneurysma der A. mesenterica superior nachweisen lässt. Im Vergleich zum abdominellen Aortenaneurysma ist das Rupturrisiko ist mit 3-9,6\% geringer, die perioperative Mortalitätsrate allerdings gleich hoch.

\section{Symptome}

Die Aneurysmen sind in den häufigsten Fällen symptomlos und werden in der abdominellen Computertomographie inzidentell entdeckt. In der Notfallsituation 
Hier steht eine Anzeige.

黛 Springer 
Es besteht eine Vielzahl an beschriebenen Bypassanschlussmöglichkeiten. Generell wird ein Eingefäßbypass mit Einstrom von der A. mesenterica superior angestrebt.

Bei einer akute Mesenterialischämie, die durch eine mesenterische Venenthrombose verursacht ist, kann eine venöse Thrombektomie in Erwägung gezogen werden. Die Erfolgsrate dieser Technik ist allerdings umstritten [11].

\section{Endovaskuläre Therapie}

Aufgrund der hochsensitiven präoperativen Diagnostik mittels CTA und MRA, die eine exakte Beschreibung der Gefäßanatomie gewährleistet, wird der endovaskuläre Therapieansatz heutzutage vermehrt angewandt [7].

\section{$>$ Bei der arteriellen Katheterisierung}

besteht die Gefahr, dass durch

den Eingriff per se eine Thrombo-

embolie provoziert werden kann.

Die Thrombolyse wurde ebenfalls mehrfach angewandt und beschrieben. Die größte Sorge im Rahmen dieser Technik besteht in der begrenzten Zeitspanne sowie in dem erhöhten Risiko für eine ischämische Darmblutung [12]. Es konnte gezeigt werden, dass die gleichzeitige Anwendung von Lyse und Thrombektomie zu einer schnelleren Thrombusentfernung führt. Die Thrombolyse schließt eine anschließende Laparotomie aus und sollte daher nur in Fällen mit ausreichender kollateraler Blutversorgung angewandt werden [12].

Die perkutane transluminale Angioplastie (PTA) wird wegen der limitierten Haltbarkeit und dem Risiko einer arteriellen Dissektion, die weitere Therapieoptionen ausschließt, nicht empfohlen [13].

\section{Hybridtherapie}

Da eine endovaskuläre Revaskularisierung aufgrund der fehlenden Darminspektion und der Gefahr der distalen Embolisation sowie Dissektion als Einzelverfahren selten angewandt wird, kommt heutzutage die sog. ROMS-Technik („retrograde open mesenteric stent") vermehrt zum Einsatz. Hierbei handelt es sich um eine Kombination aus offen chirurgischer und endovaskulärer Intervention. Bei der Ver-

Gastroenterologe 2012·7:111-120 DOI 10.1007/s11377-011-0580-7

(c) Springer-Verlag 2012

\section{F. Grubhofer · F. Pecoraro · T.S. Gilhofer · C. Gubler · G. Lurje · P.M. Schneider · M. Lachat Akute Gefäßerkrankungen in der Gastroenterologie}

\section{Zusammenfassung}

Vaskulär-gastroenterologische Notfälle zählen zu den häufigen Krankheitsbildern auf internistischen und chirurgischen Notfallstationen. Die klinischen Konsequenzen reichen von trivialen bis zu lebensbedrohlichen Situationen. Nur eine frühzeitige Erkennung der Symptomenkomplexe und die Anwendung der adäquaten diagnostischen Mittel führen zur korrekten Diagnosestellung mit nachfolgend - möglicherweise lebensrettender - Therapie. Um die hohen Mortalitätsraten der akuten Mesenterialischämien (50\%), aortoenterischen Fisteln (30-40\%), Aneurysmen viszeraler Arterien (10-100\%) sowie des
Budd-Chiari-Syndroms weiter senken zu können, gewinnen neue Strategien mit endovaskulärem Therapieansatz zunehmend an Bedeutung und ersetzen teilweise über viele Jahrzehnte etablierte Diagnose- und Therapiealgorithmen. Diese Übersichtsarbeit soll einen Überblick über aktuelle Diagnostikund Therapiekonzepte häufiger vaskulär-gastroenterologischer Notfälle verschaffen.

\section{Schlüsselwörter}

Mesenterialischämie · Aortoenterische Fistel · Viszeralarterienaneurysma - Budd-ChiariSyndrom · Endovaskuläre Therapieansätze

\section{Acute gastroenterologic vascular diseases}

Abstract

Acute gastroenterologic vascular emergencies are common situations in emergency departments and the clinical consequences range from trivial to life-threatening. Only the early recognition of these symptom patterns and prompt use of the appropriate diagnostic tools lead to a correct diagnosis with subsequent potentially life-saving treatment. To decrease the high mortality rate of acute mesenteric ischemia (50\%), aorto-enteric fistula (30-40\%), visceral artery aneurysms (10$100 \%$ ) and Budd-Chiari syndrome new strate- gies with an endovascular approach are gaining importance and are partially replacing established diagnostic and therapeutic algorithms. This article provides a review of the diagnosis and therapy of these gastroenterologic emergency situations.

\section{Keywords}

Mesenteric ischemia - Aorto-enteric fistula . Visceral artery aneurysms - Budd-Chiari syndrome - Endovascular therapy approaches wendung der ROMS-Technik konnte im Vergleich zur anterograden Bypasstechnik eine verringerte Mortalität nachgewiesen werden. Allerdings scheint die Wiederverschlussrate bei ROMS höher zu sein als bei anterogradem Bypass [14, 15]. Bei Patienten mit erhöhtem Infektionsrisiko aufgrund von Darmischämie stellt ROMS eine hilfreiche Alternative zur mesenterialen Bypasschirurgie dar [13].

\section{Mesenterialvenenthrombose}

\section{Epidemiologie und Ätiologie}

Die Mesenterialvenenthrombose ist - wie bereits oben erwähnt - eine eher seltene, aber sehr heimtückische Form der Mesenterialischämie. Die hohe Mortalitätsrate von $34 \%$ lässt sich $u$. a. durch die zeit- liche Verzögerung bis zur Diagnosestellung erklären. Das Risiko, eine Mesenterialvenenthrombose zu entwickeln, steigt bei Patienten mit Hyperkoagulabilität. Krankheitsbilder, die eine hyperkoagulabile Neigung mit sich bringen, sind z. B. die Polycythaemia vera, der Protein-Cund -S-Mangel [1], aber auch viszerale Infektionen, portale Hypertension, Hohlorganperforation, abdominelles Trauma sowie vorangegangene offene oder laparoskopische Bauchoperationen. Bei Patienten nach Splenektomie, Kolektomie oder Roux-en-Y-Bypassoperation konnte ebenfalls ein erhöhtes Risiko nachgewiesen werden. Die häufigste Ursache für eine Mesenterialvenenthrombose scheint jedoch eine intraabdominelle Sepsis zu sein $[16,17,18,19]$. 


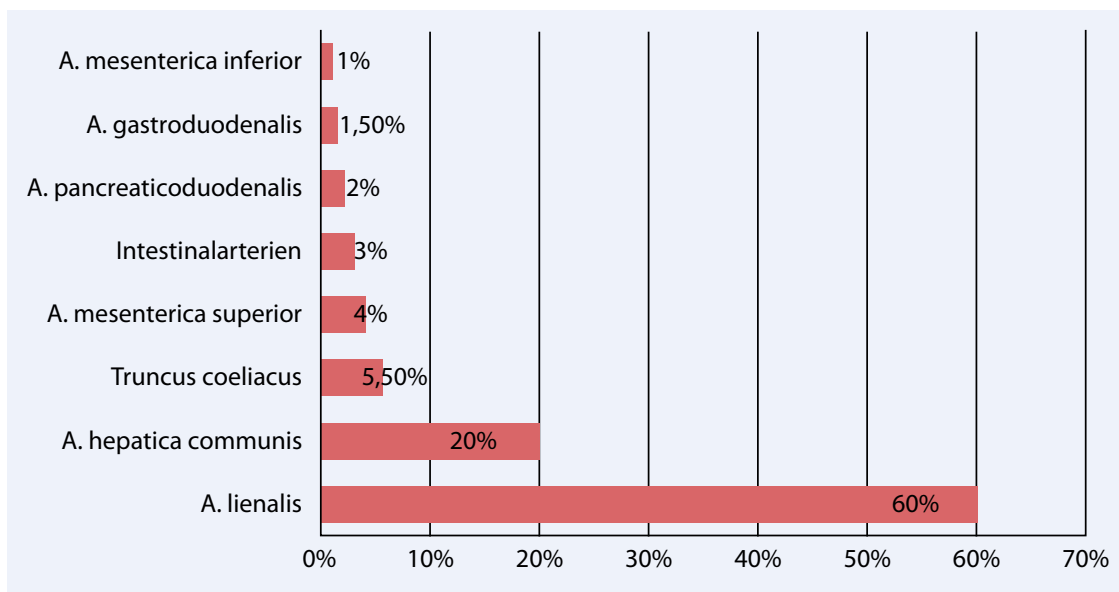

Abb. $3 \Delta$ Häufigkeit von Aneurysmen viszeraler Arterien. (Adaptiert nach [27])

zeigen AVA-Patienten Zeichen einer Hypovolämie oder diffuse Bauchschmerzen. Es gibt keine charakteristischen Symptome, die auf dieses Krankheitsbild hinweisen.

\section{Diagnostik}

Neben der klinischen und laborchemischen Untersuchung ist bei AVA-Verdacht die CTA oder die selektive digitale Subtraktionsangiographie (DSA) durchzuführen. Bei Nachweis eines AVA soll zum Ausschluss eines begleitenden zerebralen Aneurysmas eine zerebrale MRT oder CT folgen.

\section{Therapie}

In der Notfallsituation wird eine explorative Laparotomie durchgeführt. Intraoperativ wird entschieden, ob die betroffene Arterie durch eine Ligatur versorgt wird oder ob eine gefäßchirurgische Rekonstruktion durchgeführt wird.

Je nach Morphologie und Lokalisation werden verschiedene chirurgische oder endovaskuläre Interventionsmöglichkeiten in Betracht gezogen:

- Der endovaskuläre Therapieansatz mittels Arterienembolisation gilt bei sackförmigen Aneurysmen als vielversprechende minimalinvasive $\mathrm{Al}$ ternative mit einer geringeren Mortalität und Morbidität [28]. Die Erfolgsrate liegt zwischen 85 und $90 \%$.

- Die Stentimplantation wird ebenfalls angewandt, doch die kleinen Gefäßdurchmesser limitieren den Gebrauch dieser Methode.
- Liegt ein hilusnahes, nicht sackförmiges Aneurysma der Milzarterie vor, so empfiehlt sich eine Splenektomie.

- Bei einem Aneurysma der A. hepatica communis soll - wenn möglich - eine endovaskuläre Arterienembolisation durchgeführt werden. Verhindert die Morphologie die endovaskuläre Therapie, kann alternativ eine offene Aneurysmaresektion mit Gefäßrekonstruktion oder, bei ungehinderter portaler Perfusion, eine Ligatur der Leberarterie in Betracht gezogen werden.

- Aneurysmen in der A. mesenterica superior sollten durch Arterienrekonstruktion behoben werden.

Aufgrund der Vielfältigkeit in Bezug auf Ätiologie, Lokalisation und aufgrund der unterschiedlichen kollateralen Kreislaufverhältnisse ist es schwierig, einheitliche Therapiekriterien zu setzen. Grundsätzlich sollte wegen der im Vergleich zu notfallmäßigen Therapiemaßnahmen niedrigen Mortalitätsrate eine elektive Behandlung angestrebt werden.

\section{Budd-Chiari-Syndrom}

\section{Definition und Pathogenese}

Das Budd-Chiari-Syndrom stellt eine Obstruktion des venösen Abflusses der Leber auf Höhe der (intra)hepatischen Venolen, der großen Lebervenen, der V. cava inferior (intravasal durch Thromben oder von extern durch vergrößerten Lobus caudatus) oder des rechten Vor- hofs dar. Aufgrund des daraus resultierenden erhöhten intrahepatischen sinusoidalen Drucks kommt es im Verlauf zu einer portalen Hypertension, die im Rahmen der verringerten portalen Perfusion der Leber zu Pfortaderthrombosen führen kann. Die Kombination von Pfortaderthrombosen und nachfolgendem Freisetzen von freien Radikalen zeigt im Vollbild eines Budd-Chiari-Syndroms hypoxische Schäden im Sinne einer Leberzellnekrose und Leberzirrhose. Prädisponierende Faktoren bzw. Auslöser eines Budd-Chiari-Syndroms sind in 75\% der Fälle angeborene oder erworbene Hyperkoagulabilität ([29], • Tab. 1).

\section{Symptome}

Die Klinik ist abhängig vom Zeitraum, in dem sich die Okklusion der hepatovenösen Abflusswege entwickelt und dementsprechend davon, ob sich ein venöser Kollateralkreislauf ausgebildet hat. Die akute Form geht mit Übelkeit, Erbrechen, Ikterus, Aszites und schlimmstenfalls mit einer hepatischen Enzephalopathie einher, während die häufigere subakute Form einen schleichenden Beginn aufweist und mit nur minimalen Hepatozytennekrosen und Aszites verbunden ist. Die chronische Form stellt meist die Komplikation einer Zirrhose dar und geht mit Splenomegalie sowie ösophagogastralen Varizen einher. Bauchschmerzen, Hepatomegalie und Aszites können bei allen Formen auftreten. Bei okkludierter V. cava inferior bilden sich oft Beinödeme und sichtbar dilatierte Kollateralvenen an der Flanke sowie am Rücken aus.

\section{Diagnostik}

In der akuten Form des Budd-Chiari-Syndroms sind die Transaminasen bis über die 5-fache Norm erhöht, während sie bei der subakuten Ausprägung nur leicht erhöht sein können. Alkalische Phosphatase und Bilirubin steigen verzögert an, Albumin ist vielfach erniedrigt. Der Serum-AszitesAlbumin-Gradient ist wie beim kardiogenen Aszites hoch, das Gesamtprotein im Aszites beträgt normalerweise $<2,5 \mathrm{~g} / \mathrm{dl}$. Das diagnostische Mittel der Wahl stellt die Dopplersonographie dar (Sensitivität und Spezifität $>85 \%$ ). 


\begin{tabular}{|ll}
\hline Tab. 1 Mögliche Ursachen des Budd-Chiari-Syndroms. (Nach [29]) \\
\hline Vererbte Grunderkrankungen & Erworbene Ursachen \\
\hline - Antithrombin-III-Mangel & - Myeloproliferatives Syndrom \\
- Protein-C-Mangel & - Polycythaemia vera \\
- Protein-S-Mangel & - Essenzielle Thrombozythämie \\
- Faktor-V-Leiden-Mutation & - Myelofibrose \\
- Prothrombin-Mutationen & - Paroxysmale nächtliche Hämoglobinurie \\
& - Antiphospholipidsyndrom \\
& - Einnahme oraler Kontrazeptiva \\
& - Schwangerschaft \\
\hline
\end{tabular}

Die Computertomographie wird oft eingesetzt, wenn eine TIPS-Einlage erwogen wird (s. unten), da nekrotische Areale im kontrastmittelunterstützten CT besser sichtbar sind. Das MRT weist Vorteile auf in der Differenzierung zwischen akuter, subakuter und chronischer Form des Budd-Chiari-Syndroms [30].

$>$ Gesichert wird die Diagnose

durch eine "Spinnwebenform"

in der Venographie der Leber.

Die Messung des portokavalen Druckgradienten ist hilfreich bei Frage, ob eine Shuntimplantation sinnvoll ist. Im Rahmen einer diagnostischen Angiographie sollten wenn möglich noch Biopsien des rechten und linken Leberlappens gemacht werden.

\section{Therapie}

Es gilt primär, die hepatovenöse Thrombose durch Antikoagulation mittels kurzfristiger Heparin-Gabe in ihrer Ausbreitung zu bremsen. Grundsätzlich kann bei symptomarmen Patienten mit wenig ausgeprägtem Leberfunktionsdefizit und fehlenden Hinweisen auf eine Lebergewebsnekrose eine medikamentöse Therapie alleine gerechtfertigt werden. Bei fulminanten Verläufen mit Enzephalopathie und hepatorenalem Syndrom oder bei manifester Lebernekrose muss eine interventionelle oder chirurgische Behebung der hepatovenösen Abflussbehinderung angestrebt werden.

\section{Thrombolyse und Angioplastie}

Bei Patienten mit frischer Thrombusbildung kann die Thrombolyse mittels Urokinase oder Plasminogenaktivatoren direkt in die thrombosierte Lebervene über einen transjugulären oder transfemoralen Venenkatheter eingesetzt werden. Eine Symptomverbesserung wurde bei $70 \%$ der Patienten erzielt, die mittels perkutaner transluminaler Angioplastie therapiert wurden [30]. Hierfür eignen sich Patienten mit kurzen Stenosen in der V. cava inferior oder segmentalen Stenosen in den Lebervenen. Die relativ hohen Rezidivraten verlangen engmaschige Dopplersonographiekontrollen.

\section{Transjugulärer intrahepatischer Shunt}

Der transjuguläre intrahepatische portosystemische Shunt (TIPS) wird bei Patienten angewandt, bei denen sowohl konservative Therapie als auch Lysetherapie versagt haben. Weitere Indikationen sind das Vorliegen eines thrombotischen Verschlusses der V. cava inferior, eine stark reduzierte Leberfunktion oder ein portokavaler Druckgradient unter $10 \mathrm{mmHg}$. Es wird primär eine Shuntverbindung zwischen einer V. hepatica und der V. portae angestrebt. Kann aufgrund einer Thrombose oder wegen diffizilen anatomischen Verhältnissen keine hepatische Vene verwendet werden, so kann der Shunt auch mit der retrohepatischen V. cava inferior und der V. portae gebildet werden. Dieser Shunt hat zwar eine hohe Tendenz zu verschließen, gewährt dem Organismus allerdings Zeit, kollaterale Kreisläufe zu bilden. Der TIPS wird häufig auch als Überbrückungstherapie vor der Lebertransplantation verwendet.

\section{Chirurgie}

Als chirurgische Therapieoptionen stehen die Anlage eines portosystemischen Shunts, die Lebertransplantation und in den seltensten Fällen die Senning-Operation (hepatoatriale Anastomose) zur Ver- fügung. Für eine portosystemische Shuntanlage qualifizieren vor allem Patienten mit leichter Lebererkrankung (ChildPugh A) und niedrigem MELD-Score („Model of End-Stage Liver Disease“), also Patienten mit subakuter Verlaufsform des Budd-Chiari-Syndroms, bei denen die verursachende Grunderkrankung ein gutes Ergebnis erwarten lässt (z. B. Polycythaemia vera, essenzielle Thrombozythämie).

Bei fortgeschrittener Lebererkrankung (Child-Pugh B oder C, hoher MELDScore) steigt die operationsassozierte Mortalitätsrate signifikant an. Besteht bei diesen Patienten eine Grunderkrankung mit günstiger Lebenserwartung, so kann eine Lebertransplantation in Betracht gezogen werden. Obwohl membranöse kavale Gefäßobstruktionen hauptsächlich mittels Angioplastie behandelt werden, wurde in vereinzelten Fällen eine Senning-Operation durchgeführt, bei welcher der venöse hepatische Ausfluss direkt mit dem rechten Vorhof anastomosiert wird.

\section{Fazit für die Praxis}

- Die beschriebenen vaskulären Erkrankungen stellen gastroenterologische Notfallsituationen dar, die eine sofortige Abklärung und Therapie verlangen.

- Die endoskopische und die endovaskuläre - computertomographisch unterstützte - Diagnostik erweisen sich als Mittel der Wahl und sollten mit niedriger Hemmschwelle in den diagnostischen Algorithmus einbezogen werden. In einzelnen Studien wurde bereits nachgewiesen, dass der frühzeitige Einsatz endoskopischer und endovaskulärer Diagnostik die Kosten reduzieren hilft [31].

- Je nach Befund erfordert die Therapie zumeist eine interdisziplinäre Zusammenarbeit. Die behandelnden Mediziner sind aufgefordert, frühzeitig den Zeitpunkt zu erkennen, wann die Hilfe anderer Fachdisziplinen mit einbezogen werden sollte. 
Hier steht eine Anzeige.

黛 Springer 


\section{Korrespondenzadresse}

Prof. Dr. M. Lachat

Klinik für Herz- und Gefässchirurgie,

UniversitätsSpital Zürich

Rämistr. 100, 8091 Zürich

Schweiz

Mario.lachat@usz.ch

Interessenkonflikt. Der korrespondierende Autor gibt für sich und seine Koautoren an, dass kein Interessenkonflikt besteht.

\section{Literatur}

1. Roobottom CA, Dubbins PA (1993) Significant disease of the celiac and superior mesenteric arteries in asymptomatic patients: predictive value of Doppler sonography. Am J Roentgenol 161: 985988

2. Acosta S, Wadman M, Syk I et al (2010) Epidemiology and prognostic factors in acute superior mesenteric artery occlusion. J Gastrointest Surg 14: 628-635

3. Lock G (2001) Acute intestinal ischaemia. Best Pract Res Clin Gastroenterol 15: 83-98

4. Howard TJ, Plaskon LA, Wiebke EA et al (1996) Nonocclusive mesenteric ischemia remains a diagnostic dilemma. Am J Surg 171: 405-408

5. Boley SJ, Feinstein FR, Sammartano R et al (1981) New concepts in the management of emboli of the superior mesenteric artery. Surg Gynecol Obstet 153: 561-569

6. Taourel PG, Deneuville M, Pradel JA et al (1996) Acute mesenteric ischemia: diagnosis with contrast-enhanced CT. Radiology 199: 632-636

7. Aschoff AJ, Stuber G, Becker BW et al (2009) Evaluation of acute mesenteric ischemia: accuracy of biphasic mesenteric multi-detector $\mathrm{CT}$ angiography. Abdom Imaging 34: 345-357

8. Acosta S, Ogren M, Sternby NH et al (2004) Incidence of acute thrombo-embolic occlusion of the superior mesenteric artery-a population-based study. Eur J Vasc Endovasc Surg 27: 145-150

9. Mamode N, Pickford I, Leiberman P (1999) Failure to improve outcome in acute mesenteric ischaemia: seven-year review. Eur J Surg 165: 203-208

10. Wyers MC (2010) Acute mesenteric ischemia: diagnostic approach and surgical treatment. Semin Vasc Surg 23: 9-20

11. Bergqvist D, Svensson PJ (2010) Treatment of mesenteric vein thrombosis. Semin Vasc Surg 23: 65-

12. Acosta S, Sonesson B, Resch T (2009) Endovascular therapeutic approaches for acute superior mesenteric artery occlusion. Cardiovasc Intervent Radiol 32: 896-905

13. Resch TA, Acosta S, Sonesson B (2010) Endovascular techniques in acute arterial mesenteric ischemia. Semin Vasc Surg 23: 29-35

14. Milner R, Woo EY, Carpenter JP (2004) Superior mesenteric artery angioplasty and stenting via a retrograde approach in a patient with bowel ischemia-a case report. Vasc Endovascular Surg 38: 8991

15. Wyers MC, Powell RJ, Nolan BW, Cronenwett JL (2007) Retrograde mesenteric stenting during laparotomy for acute occlusive mesenteric ischemia. J Vasc Surg 45: 269-275
16. Wang MQ, Lin HY, Guo LP et al (2009) Acute extensive portal and mesenteric venous thrombosis after splenectomy: treated by interventional thrombolysis with transjugular approach. World J Gastroenterol 15: 3038-3045

17. Stamou KM, Toutouzas KG, Kekis PB et al (2006) Prospective study of the incidence and risk factors of postsplenectomy thrombosis of the portal, mesenteric, and splenic veins. Arch Surg 141:663669

18. James AW, Rabl C, Westphalen AC et al (2009) Portomesenteric venous thrombosis after laparoscopic surgery: a systematic literature review. Arch Surg 144: 520-526

19. Swartz DE, Felix EL (2004) Acute mesenteric venous thrombosis following laparoscopic Rouxen-Y gastric bypass. JSLS 8: 165-169

20. Acosta S, Ogren M, Sternby NH et al (2005) Mesenteric venous thrombosis with transmural intestinal infarction: a population-based study. JVasc Surg 41: 59-63

21. Saers SJ, Scheltinga MR (2005) Primary aortoenteric fistula. Br J Surg 92: 143-152

22. Baril DT, Carroccio A, Ellozy SH et al (2006) Evolving strategies for the treatment of aortoenteric fistulas. J Vasc Surg 44: 250-257

23. Sweeney MS, Gadacz TR (1984) Primary aortoduodenal fistula: manifestation, diagnosis, and treatment. Surgery 96: 492-497

24. Armstrong PA, Back MR, Wilson JS et al (2005) Improved outcomes in the recent management of secondary aortoenteric fistula. J Vasc Surg 42: 660-666

25. Burks JA Jr, Faries PL, Gravereaux EC et al (2001) Endovascular repair of bleeding aortoenteric fistulas: a 5-year experience. J Vasc Surg 34: 1055-1059

26. Eskandari MK, Resnick SA (2005) Aneurysms of the renal artery. Semin Vasc Surg 18: 202-208

27. Pulli R, Dorigo W, Troisi N et al (2008) Surgical treatment of visceral artery aneurysms: A 25-year experience. J Vasc Surg 48: 334-342

28. Ruiz-Tovar J, Martinez-Molina E, Morales V et al (2007) Evolution of the therapeutic approach of visceral artery aneurysms. Scand J Surg 96: 308313

29. Mitchell MC, Boitnott JK, Kaufman S et al (1982) Budd-Chiari syndrome: etiology, diagnosis and management. Medicine (Baltimore) 61: 199-218

30. Yang XL, Cheng TO, Chen CR (1996) Successful treatment by percutaneous balloon angioplasty of Budd-Chiari syndrome caused by membranous obstruction of inferior vena cava: 8-year follow-up study. J Am Coll Cardiol 28: 1720-1724

31. Lee JG, Turnipseed S, Romano PS et al (1999) Endoscopy-based triage significantly reduces hospitalization rates and costs of treating upper GI bleeding: a randomized controlled trial. Gastrointest Endosc 50: 755-761

\section{Mögliche Alternative zu Antibiotika entwickelt}

Als Ursache für die wachsende Verbreitung resistenter humanpathogener Bakterien nennt die Weltgesundheitsorganisation WHO den unsachgemäßen Einsatz von Penicillin und $\mathrm{Co}$.

Forscher des Fraunhofer-Instituts für Zelltherapie und Immunologie IZI in Leipzig haben eine mögliche Alternative zu den etablierten Antibiotika gefunden: Antimikrobielle Peptide bestehend aus weniger als 20 Aminosäuren. Aus bekannten fungizid und bakterizid wirkenden Peptiden erzeugten die Forscher zunächst Sequenzvariationen und testeten diese in vitro an unterschiedlichen Keimen. Neben Enterokokken, Hefen und Schimmelpilzen wurden auch humanpathogene Bakterien wie Streptococcus mutans abgetötet. Der multiresistente Krankenhauskeim Staphylococcus aureus wurde in seinem Wachstum stark beeinträchtigt. Die neu erzeugten Peptide besitzen kationische Aminosäurereste und können dadurch an die negativ geladene Bakterienmembran binden und diese durchdringen. Das Wirkspektrum der untersuchten Peptide schließt neben Bakterien und Pilzen auch lipidumhüllte Viren ein. Gleichzeitig sind die identifizierten Peptide unschädlich für gesunde Körperzellen. Im nächsten Schritt wollen die Forscher die antimikrobiellen Peptide in vivo an Infektionsmodellen testen.

Quelle:

Fraunhofer-Institut für Zelltherapie und Immunologie, Leipzig, www.izi.fraunhofer.de 